\title{
ENSAIO DE COMPRESSÃO E MODELO MATEMÁTICA DO COMPÓSITO AISI 316L/SYCRO
}

\author{
J. F. Salvador ${ }^{1,3}$, A. S. Cavichin ${ }^{2}$, \\ ${ }^{1}$ Departamento de Engenharia Mecânica, Instituto Federal do Espírito Santo, Vitória - Brasil. \\ ${ }^{2}$ Pós-Graduação em Oceanografia Ambiental, Universidade Federal do Espírito Santo - Brasil. \\ ${ }^{3}$ Pós-Graduação em Engenharia Mecânica, Universidade Federal do Espírito Santo - Brasil
}

\section{RESUMO}

$\mathrm{Na}$ confecção de possíveis produtos magnéticos a partir cerâmico $\mathrm{Sr}_{1.8} \mathrm{Y}_{0.2} \mathrm{CrReO}_{6}$ (SYCRO), como sensores atuadores e artigos de spintrônica serão necessário melhorar a resistência mecânica com o uso dos compósitos para sua proteção química e mecânica. No entanto precisa-se verificar a resistência de tal compósito sem afetar suas características. Dessa forma, realizamos testes de compressão mecânica e modelagem matemática. O compósito apresentou um comportamento mecânico de esponja mantendo sua integridade após ensaio.

\section{ABSTRACT}

In the preparation of possible magnetic products from $\mathrm{Sr}_{1.8} \mathrm{Y}_{0.2} \mathrm{CrReO}_{6}$ (SYCRO) ceramic, such as actuating sensors and spintronic articles, it will be necessary to improve the mechanical resistance with the use of composites for their chemical and mechanical protection. However, it is necessary to verify the strength of such composite without affecting its characteristics. Thus, we performed tests of mechanical compression and mathematical modeling. The composite presented a sponge mechanical behavior maintaining its integrity after the test.

\section{I- INTRODUÇÃO}

Para os futuros produtos que poderão ser desenvolvidos a base de cerâmica $\mathrm{Sr}_{1.8} \mathrm{Y}_{0.2} \mathrm{CrReO}_{6}$ (SYCRO) [1], desenvolvido em nosso laboratório, que apresenta propriedades magnética "mole" de grande interesse para confecção de dispositivos diversos como sensores, atuadores, e aplicações de spintrônica. No entanto a resistência mecânica deste cerâmico não satisfatória e nem sua resistência a umidade e meios ácidos, acreditamos ser devida presença do $\mathrm{SrO}$ na formulação do composto SYCRO [1].

Para solucionar tal problema, estamos sugerindo o uso por meio de compósitos material inox, aço $316 \mathrm{~L}$ que amplas aplicações industriais e biomédicas. O compósito com matriz de AISI $316 \mathrm{~L}$ poderá conferir resistência química e mecânica, além de biocompatibildade.

Para certificar esta resistência mecânica foram feitos teste de compressão no compósito a partir do compósito obtido e uma amostra de 316 L para referência de comparação. Também foram feitas medidas de massas antes e depois do experimento.

foi adaptado um modelo matemático de esponja descrito por Avalle et al. (Equação 1) [2], Peroni et al. Eq (2) [3]: 


\section{II- MATERIAIS E MÉTODOS:}

O ensaio de compressão é feito com a aplicação de carga compressiva uniaxial uniforme na seção transversal em um CDP (corpo de prova), onde a deformação linear obtida pela medida da distância entre as placas que comprimem o corpo versus a carga de compressão consiste no resultado do ensaio. O ensaio de compressão seguiu a norma E9-09 para ensaios de compressão de materiais metálicos em temperatura ambiente [4].

\section{III- RESULTADOS}

As fotos das amostras comprimidas encontram-se na figura 01. Pode ser observado que ocorreu uma redução no volume da amostra.

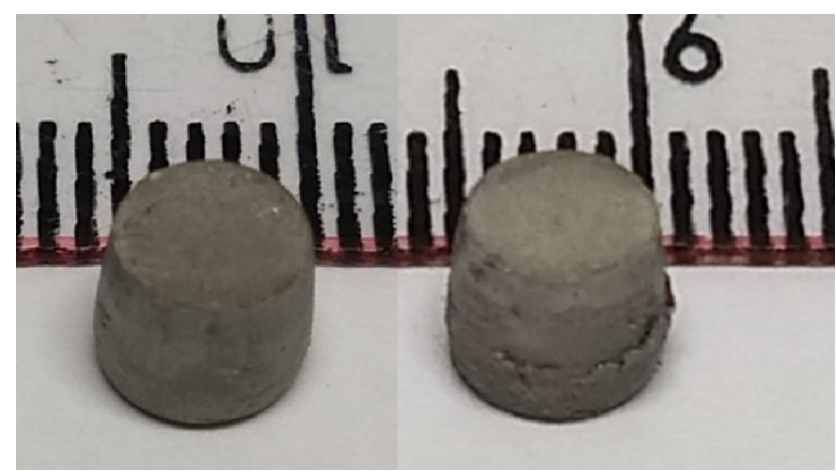

Figura 01 fotos montadas lado a lado após ensaio de compressão, sendo a amostra da esquerda a amostra 316L/SYCRO, e à direita a foto da amostra de referência $316 \mathrm{~L}$ após ensaio de compressão. Fonte o autor.

As curvas obtidas no ensaio de compressão são mostradas na figura 02 Figura . Não houve variação de massa significativa de ambas as amostras após o ensaio. Foi observada uma recuperação elástica de aproximadamente 12\% e 16\% para a amostra 316L e 316L/SYCRO, respectivamente. A amostra 316L mostrou trincas e falhas aparentes após o ensaio. Os valores obtidos encontram-se na Tabela I.

Tabela I - Massa, diâmetros médios, densidade aparente e densidade teórica das amostras 316L e 316L/SYCRO. Os valores em parênteses representam as incertezas na última casa decimal.

\begin{tabular}{|l|l|l|l|l|l|}
\hline Amostra & Massa (g) & $\mathbf{D}_{\mathbf{0}}(\mathbf{m m})$ & $\mathbf{L}_{\mathbf{0}}(\mathbf{m m})$ & $\boldsymbol{\rho}_{\mathbf{0}}\left(\mathbf{g} / \mathbf{c m}^{\mathbf{3}}\right)$ & $\boldsymbol{\rho}_{\text {teo }}\left(\mathbf{g} / \mathbf{c m}^{\mathbf{3}}\right)$ \\
\hline $316 \mathrm{~L}$ & $0,8883(1)$ & $4,9(1)$ & $8,3(3)$ & $5,5(3)$ & $7,884(1)$ \\
\hline $316 \mathrm{~L} /$ SYCRO & $0,8816(1)$ & $4,9(3)$ & $8,1(3)$ & $5,8(3)$ & $7,807(1)$ \\
\hline
\end{tabular}

A análise da densidade calculada pela $\mu$ TC apresentou uma redução de $17 \%$ em relação a densidade ideal do compósito. A diferença entre a densidade ideal e a calculada sugere que $17 \%$ do compósito é formado por poros menores que $5 \mu \mathrm{m}$ não detectáveis na $\mu \mathrm{TC}$. Logo, sugerimos que a resposta mecânica de ambas as amostras apresenta uma característica típica de compostos tipo esponja ("foam"), o que entendemos estar associado à microporosidade presenta nas amostras. 


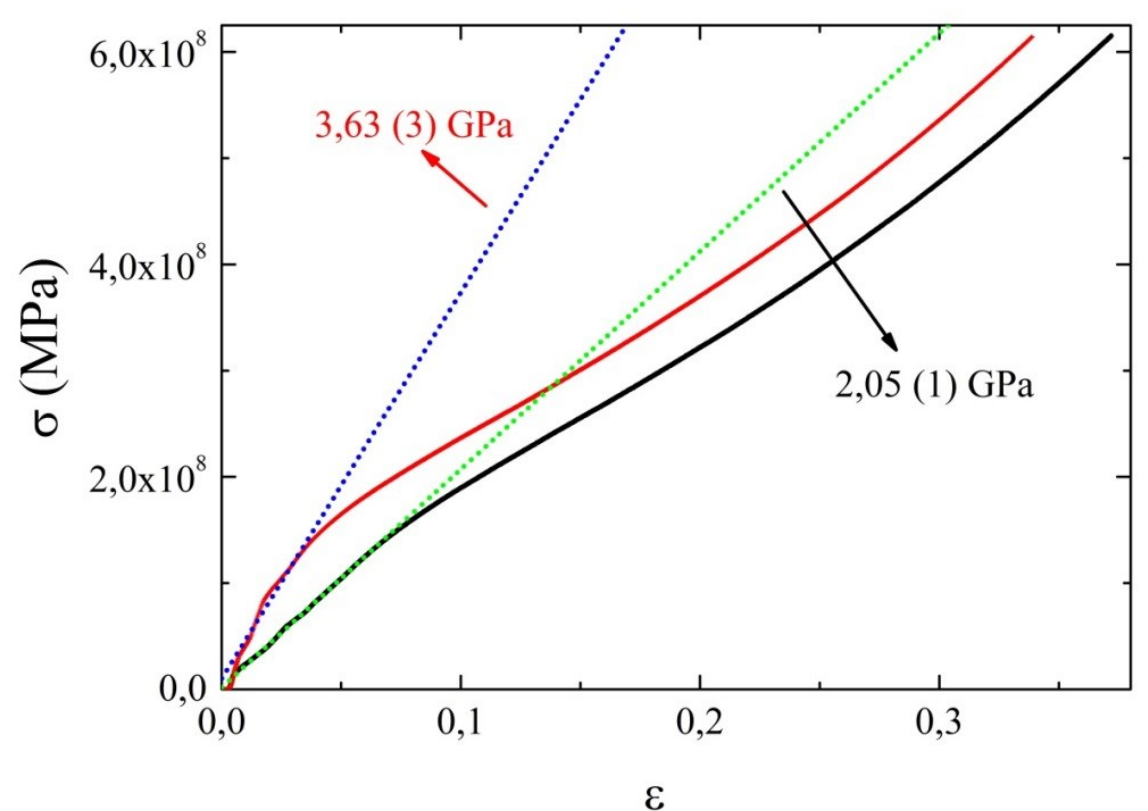

Figura 02 - Resultado do ensaio de compressão no equipamento EMIC (força $x$ deformação) com o ajuste linear para a região abaixo de $5 \%$ de deformação. A carga máxima aplicada em ambos os corpos de prova foram idênticas ( $F_{\max }=11600 \mathrm{~N}$ ).

Para determinar o Módulo de Young $(E)$ das amostras 316L e 316L/SYCRO foi ajustado, nas curvas de compressão mecânica obtidas, funções lineares do tipo $\sigma=E \epsilon+\sigma_{0}$ em duas regiões distintas, abaixo e acima de $5 \%$ de deformação. As retas obtidas no ajuste são mostradas na Figura 02 e Figura 03.

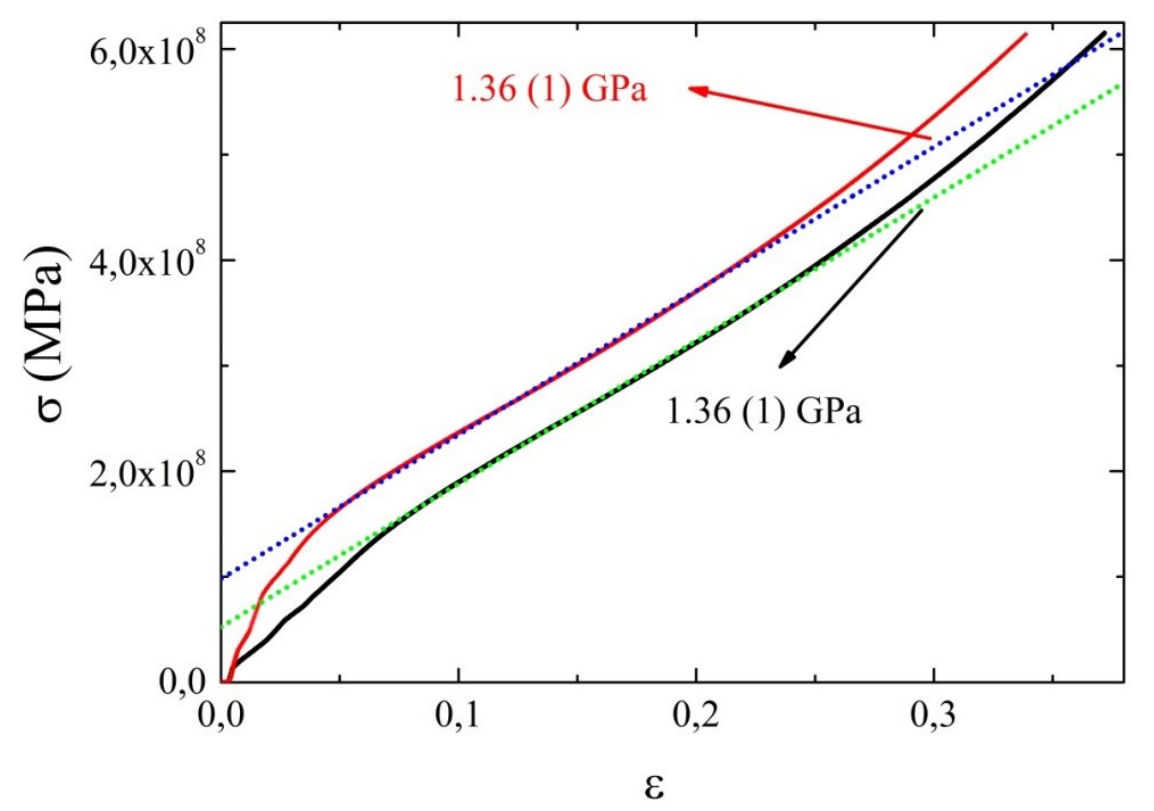

Figura 03- Resultado do ensaio de compressão no equipamento EMIC (força $x$ deformação) com o ajuste linear para a região acima de $5 \%$ de deformação. A carga máxima aplicada em ambos os corpos de prova foram idênticas $\left(F_{\max }=11600 \mathrm{~N}\right)$. 
A primeira região ajustada é considerada a região elástica e foi encontrado valores para o Módulo de Young de 2,05 \pm 0,01 GPa e 3,63 \pm 0,03 GPa para as amostras 316L e 316L/SYCRO, respectivamente. A segunda região é considerada a região plástica. Para esta região obtivemos um mesmo $E$ para ambas as amostras, de 1,36 $\pm 0,01$ GPa. Os valores obtidos em ambas as regiões são duas ordens de grandeza menor que o módulo de elasticidade encontrado na literatura para o aço AISI 316L, que é de 190 GPa. Esta diminuição faz com que o material seja mais maleável em relação ao aço de referência.

Nota-se que os módulos de elasticidade de ambas as amostras têm valores próximos numericamente para a região acima de $5 \%$ de deformação. Em termos de energia de deformação, a energia empregada na amostra 316L/SYCRO sinterizada é maior que na amostra de referência, ou seja, o aumento de rigidez foi apenas na parte inicial até deformações em torno de $5 \%$.

O modelo utilizado neste trabalho foi reportado inicialmente por Avalle et al. (Equação 9) [2] para sólidos celulares e posteriormente adaptado por Peroni et al. (Equação 10) [3] para compósitos metálicos esponjosos com taxas de compressão diferentes. Os modelos são descrito por:

$$
\begin{gathered}
\sigma=A\left(1-e^{-\left(\frac{E}{A}\right) \varepsilon(1-\varepsilon)^{m}}\right)+B\left(\frac{\varepsilon}{1-\varepsilon}\right)^{p}, \\
\sigma=A\left(1-e^{-m \varepsilon}\right)+B\left(\frac{\varepsilon}{1-\varepsilon}\right)^{p},
\end{gathered}
$$

onde $\sigma$ é a tensão de engenharia, $\varepsilon$ a deformação de engenharia, $E$ é o módulo de elasticidade, $A$ e $B$ parâmetros dependentes da densidade que descrevem a região elástica+platô e a região de densificação, respectivamente. $m$ e $p$ são expoentes dos modelos e independentes da densidade, sendo o parâmetro $m$ ligado a região elástica+platô e o parâmetro $p$ ligados à região de densificação. O modelo que melhor se ajustou para a amostra sinterizada 316 foi o apresentado na Equação 10 e para o compósito 316L/SYCRO, o modelo apresentado na Equação 9. As curvas obtidas no ajuste desses modelos são mostradas para ambas as amostras na Figura 04. 


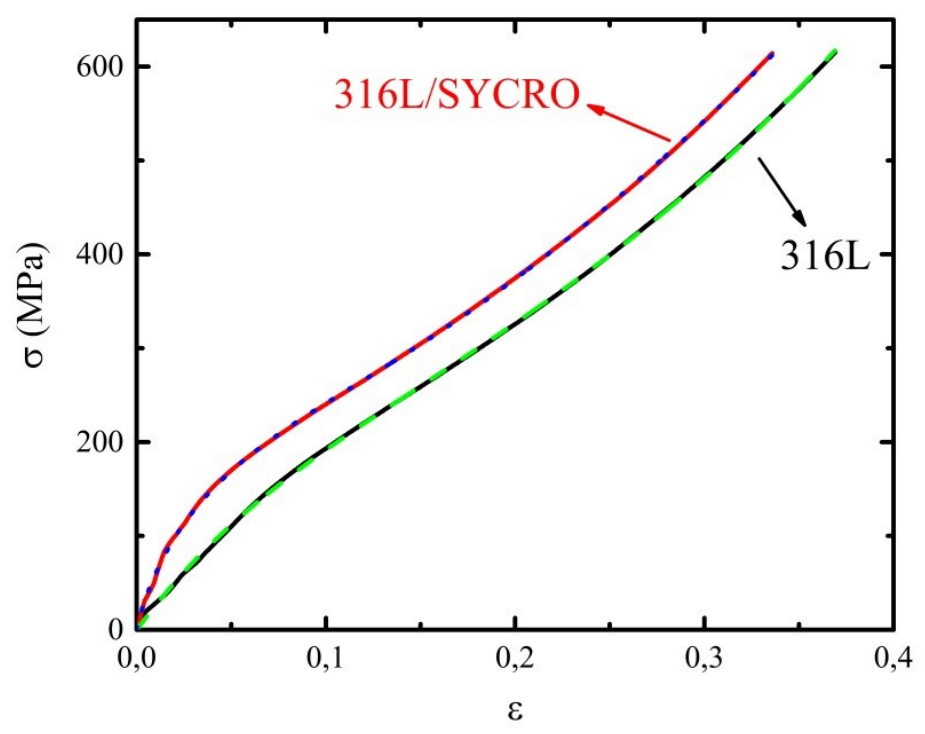

Figura 04- Modelo de esponja ajustado para as amostras de 316L e 316L/SYCRO.

Para a amostra sinterizada $316 \mathrm{~L}$, o modelo ajustou em valores de $\mathrm{A}=148 \mathrm{MPa}$ e $\mathrm{B}=842 \mathrm{MPa}$. O valor encontrado para o parâmetro $m$ foi de $m=14,9$ e o parâmetro $p$ convergiu para o valor de $p=1,1$. O compósito 316L/SYCRO, por sua vez, mostrou valores de $A=163 \mathrm{MPa}$ para e $\mathrm{B}=1,01 \mathrm{GPa}$. Os parâmetros $m$ e $p$ convergiram para valores $m=18,5$ e $p=0,7$. O módulo de elasticidade encontrado para o 316L/SYCRO foi de 3,36 GPa, valor bem próximo do encontrado pelo ajuste linear na região abaixo de $5 \%$ de deformação, o que indica um comportamento mecânico de material esponjoso.

O aumento do parâmetro $B$ (Equação 1 e 2) do compósito 316L/SYCRO em comparação ao sínter 316L indica um maior valor de energia para atingir a zona de densificação deste material. Este aumento esta relacionado a presença de um número mais elevado de micro vazios compósito 316L/SYCRO (comprovado pela $\mu \mathrm{CT}$ e pelo MEV) em relação amostra sinterizada 316L.

Considerando o parágrafo acima, destaca-se neste trabalho a facilidade de programar propriedades mecânicas e físicas (compressibilidade e magnetização) na fabricação de peças. Portanto, vemos que o material agregou propriedade magnética, melhorou as características químicas (resistência a degradação por umidade) e manteve sua característica metálica (conformabilidade).

\section{IV - CONCLUSÕES}

As amostras após o ensaio de compressão mostraram que não houve desintegração o ensaio que deformou aproximadamente $35 \%$ da sua dimensão axial, ou seja, mostrando a eficiência da integridade do compósito. O modelo de espoja representou bem o comportamento mecânico dos corpos de prova.

Notou-se que não houve variação de massa relevante durante os experimentos de compressão, ou seja, as amostras não se desfragmentaram mostrando a boa condição mecânica e estrutural dos corpos de prova (CDPs). Após o ensaio observou-se que a recuperação elástica foi de $15 \%$. 


\section{REFERENCIAS}

[1] ORLANDO, M.T.D.; CAVICHINI, A.S. ; DEPIANTI, J.B. ; PASSAMAI, J.L. ; ROCHA, J.R. ; SALVADOR, J.F. ; ORLANDO, C.G.P. . Effects of yttrium doping in ordered double perovskite Sr2CrReO6. Journal of Alloys and Compounds, v. 687, (2016), p. 463-469,.

[2] AVALLE, M.; BELINGARDI, G.; IBBA, A. Mechanical models of cellular solids: Parameters identification from experimental tests. International Journal of Impact Enginnering, 34, (2007). 3-27

[3] PERONI, L. et al. Investigation of the mechanical behaviour of AISI 316L stainless steel syntactic foams at different strain-rates. Composites: Part B, (2014). 430-442

[4] ASTM. ASTM E9-09 Standard Test Methods of Compression Testing of Metallic Materials at Room Temperature. [S.I.], p. 9. 2018. 